\title{
WestVirginiaUniversity
}

THE RESEARCH REPOSITORY @ WVU

Graduate Theses, Dissertations, and Problem Reports

2006

\section{Pair distribution functions in molecular dynamics simulations of interfaces}

Deng Cao

West Virginia University

Follow this and additional works at: https://researchrepository.wvu.edu/etd

\section{Recommended Citation}

Cao, Deng, "Pair distribution functions in molecular dynamics simulations of interfaces" (2006). Graduate Theses, Dissertations, and Problem Reports. 2352.

https://researchrepository.wvu.edu/etd/2352

This Thesis is protected by copyright and/or related rights. It has been brought to you by the The Research Repository @ WVU with permission from the rights-holder(s). You are free to use this Thesis in any way that is permitted by the copyright and related rights legislation that applies to your use. For other uses you must obtain permission from the rights-holder(s) directly, unless additional rights are indicated by a Creative Commons license in the record and/ or on the work itself. This Thesis has been accepted for inclusion in WVU Graduate Theses, Dissertations, and Problem Reports collection by an authorized administrator of The Research Repository @ WVU. For more information, please contact researchrepository@mail.wvu.edu. 


\title{
PAIR Distribution FunCTIONS IN
}

\section{MOLECUlAR DYNAMICS SimUlationS OF INTERFACES}

\author{
by \\ Deng Cao
Thesis submitted to the Eberly College of Arts and Sciences at West Virginia University
In partial fulfillment of the requirements for the degree of

\author{
Master of Science \\ in \\ Physics
}

\section{Approved by}

M. E. Bachlechner, Committee Chairperson

T. H. Myers

L. Golubović

\section{Department of Physics}

\author{
Morgantown, West Virginia \\ 2006
}

\section{Keywords: Molecular Dynamics Simulations, Pair Distribution Function Copyright 2006 Deng Cao}




\author{
Abstract \\ Pair Distribution Functions in \\ Molecular Dynamics Simulations of Interface \\ by Deng Cao
}

Thin films of silicon nitride on silicon are well suited materials for many applications including photovoltaics. Large-scale molecular-dynamics simulations of silicon/silicon nitride interfaces under externally applied tensile strain are performed in an attempt to improve understanding of this interface. The simulations reveal stress release in form of fracture, slip, pit formation, and interface phase transition under high stress condition. The silicon/silicon nitride interface is described as an eight-component system thereby offering valuable information in some of the thirty-six different pair distribution functions. We find that fracture in silicon nitride, with a centerpiece breaking off the sides, is reflected in a return to the original height of the first peak of the Si-N pair distribution function indicating that this centerpiece is essentially unstretched. Slip and pit formation in silicon as well as formation of domains of two different interface phases are identified by additional peaks in the pair distribution functions at and across the interface. Understanding selective pair distribution functions calculated at various stages of a particular simulation offer the opportunity to analyze structural and mechanical failure of large systems without knowing the detailed properties of individual atoms in the system. In particular, the occurrence of peaks reflecting new interatomic distances allows early predictions of failure. 


\section{ACKNOWLEDGMENTS}

I wish to thank my advisor, Dr. Martina E. Bachlechner, for having the energy and motivation to train me in both research and personality. She showed me the importance and elegance of doing research. And she also showed me the nature of pair distribution functions, as well as the nature of other background theory and calculations. It brings me pleasure to thank Jarrod Schiffbauer for informative discussions. These discussions are also good training for my spoken English. I am glad to thank Eric Guffey, he did the data collection and graphing for my research in summer 2005. 


\section{TABLE OF CONTENTS}

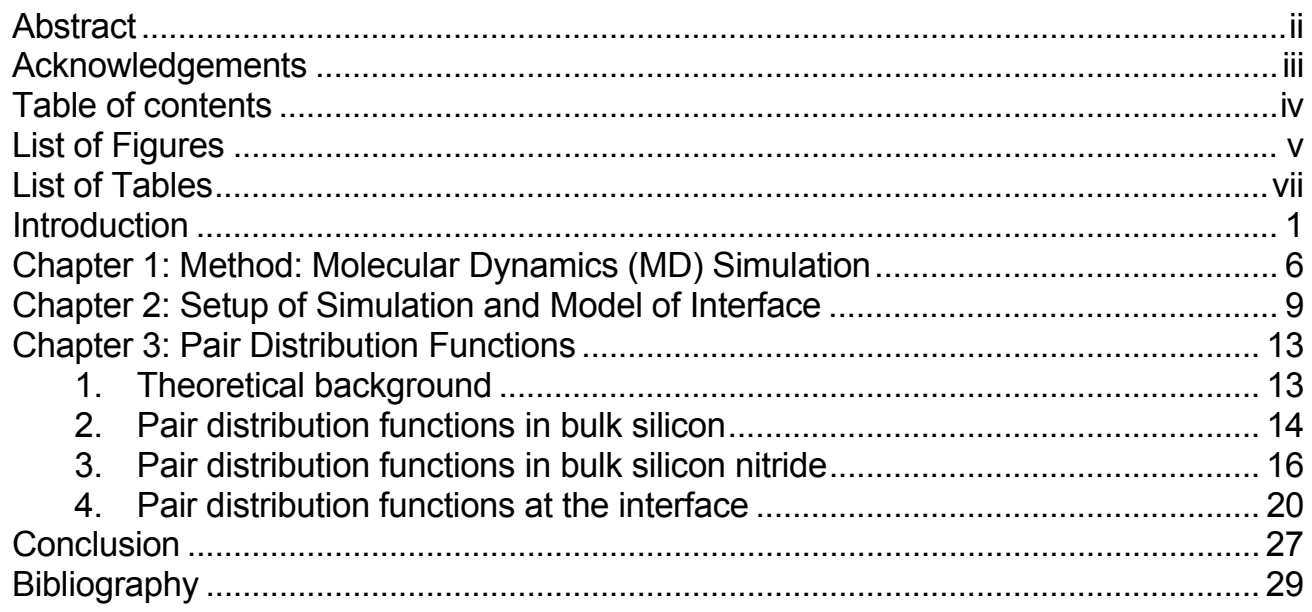




\section{LIST OF FIGURES}

Figure 1: Low-temperature passivated bifacial p-n junction Si solar cell system. Schematics taken from Aberle and Hezel, 1996.

Figure 2: Total radial distribution function of sapphire obtained from simulation (solid line) and from the x-ray diffraction studies (dotted line). Taken from Rambaut et al., 1998

Figure 3: Evolution of the unsymmetrical pair distribution $\rho^{[2]}(r ; z)$ across the shock layer.Taken from Zhakhovskii et al., 1999.

Figure 4: a): Si-N pair-distribution functions in the interior of nanoclusters (dashed lines) and in interfacial regions (solid lines) of the nanophase system. The inset figure shows the first peaks in the pair-distribution functions. b): Spatial distribution of the average $\mathrm{Si}$ coordination, projected onto the x-y plane. Taken from Kalia et al., 1997a.

Figure 5: System structure of $\mathrm{Si}(111) / \mathrm{Si} 3 \mathrm{~N} 4(0001)$ interface. Yellow spheres represent silicon, red and blue spheres represent silicon nitride. In silicon the interface, pentagons denote $\mathrm{Si}$ atoms of type 7 and squares denote Si atoms of type 8 . In silicon nitride at the interface, circles denote $\mathrm{N}$ atoms of type 4 , triangles denote $\mathrm{N}$ atoms of type 5 , and hexagons denote $\mathrm{Si}$ atoms of type 2 .

Figure 6: Atomic positions for bulk silicon in its diamond structure. (a) Conventional unit cell. Only the atoms on the top, right side, and front faces are displayed as well as the atom along the cube diagonal. (b) View onto a (111) plane.

Figure 7: Si-Si pair distribution function $g(6,6)$ in bulk silicon at different temperatures

Figure 8: Sample for geometrical calculation of theoretical nearest neighbor distances within a $\mathrm{Si}_{3} \mathrm{~N}_{4}$ unit. According to this figure the 3rd and 10th nearest neighbor distances can be estimated. Similar approach is applied for other nearest neighbors.

Figure 9: (a) Si-N pair distribution function in bulk silicon nitride at $300 \mathrm{~K}$. (b) A magnified picture from (a). The arrows indicate the peak positions (see also table 3).

Figure 10: Si-N pair distribution function in bulk silicon nitride under different strains at $600 \mathrm{~K}$.

Figure 11: Height of the first peak of $g(1,3)$ in bulk silicon nitride as depicted in figure 10 at different temperatures versus externally applied tensile strain.

Figure 12: The first 4 nearest neighbor distances in interface for $\mathrm{Si}(7)-\mathrm{Si}(7)$. 
Figure 13: The two different coherent $\mathrm{Si}(111) / \mathrm{Si}_{3} \mathrm{~N}_{4}(0001)$ interface phases. Big circles denote $\mathrm{Si}$ atoms in silicon at the interface and small circles denote $\mathrm{Si}$ and $\mathrm{N}$ atoms in the silicon nitride interface plane. Big open circles are $\mathrm{Si}(8)$ atoms, big full circles are $\mathrm{Si}(7)$ atoms. Small open circles are $\mathrm{N}(4)$ and $\mathrm{N}(5)$ atoms, small full circles are $\mathrm{Si}(2)$ atoms. (a) In phase D1, $\mathrm{N}$ atoms of type 4 in the interface plane of silicon nitride are almost directly on top of Si atoms of type 8 in the interface plane of silicon. (b) In phase D2, the silicon nitride film slipped in a way that $\mathrm{N}$ atoms of type 4 in the interface plane of silicon nitride are almost in the center of the hexagons formed by $\mathrm{Si}$ atoms at the interface.

Figure 14: Si-Si pair distribution functions $g(7,7)$ at the interface at strains of $1 \%, 14 \%$, and $28 \%$, respectively from top to bottom, and corresponding visualization of the interface silicon double layer. The insets show new peak(s) which correspond the distance suggesting a bond between two originally unbonded $\mathrm{Si}$ atoms at the interface of type 7 before original first peaks. The arrows indicate the chosen peak positions for figure 15 .

Figure 15: Height of the first peak(s) of $g(7,7)$ at silicon interface versus strain rates at different temperatures. Main line(s) starting from zero strain denote the original first peak positions. For $300 \mathrm{~K}$, we mark two different first peak positions at each specific strain rate. For $600 \mathrm{~K}$ and $900 \mathrm{~K}$, we choose the highest point at the top of first peak (as depicted in figure 14) as first peak positions. An additional line starts at $17 \%$ for all three temperatures denote the new peak positions as indicated by arrows in insets in figure 14. Height of the first peak(s) of $g(7,7)$ at silicon interface (as depicted in figure 14) versus strain rates at different temperatures.

Figure 16: Si-N pair distribution functions $g(4,8)$ across the interface at three different values of strain: (a) $8 \%$, (b) $9 \%$, and (c) $10 \%$

Figure 17: Height of the first (double) peak(s) of $g(4,8)$ across the interface (as depicted in figure 16) at different temperatures. The second peak(s) emerges at $9 \%$ for all three temperatures.

Figure 18: Visualization of the silicon/silicon nitride interface at an externally applied tensile strain of $23 \%$ strain depicting the two cracks in silicon nitride and the slip and pit in silicon.

(a) Entire system. (b) Slice in y-direction to better convey the features discussed. .26 


\section{LIST OF TABLES}

Table 1: Detailed MD simulation system description.

.12

Table 2: Comparison of geometrical and computational values of the first 4 nearest neighbor distances in bulk silicon at $300 \mathrm{~K}$. Geometric values are calculated from ideal $1^{\text {st }}$ nearest neighbor distances and ideal bond angles using law of cosine.

Table 3: Comparison of geometrical and computational values of the first 10 nearest neighbor distances in silicon nitride pairs.

Table 4: Comparison of geometrical and computational values of the first 4 nearest neighbor distances in $\mathrm{Si}(7)-\mathrm{Si}(7)$ in interface silicon. Geometric values are calculated from ideal $1^{\text {st }}$ nearest neighbor distances and ideal bond angles using law of cosine. 


\section{INTRODUCTION}

Advanced silicon solar cell technology is becoming more important as we realize that fossil energy sources are not inexhaustible. Different solar cell designs are being investigated by experiment in order to enhance efficiency and reliability. Reliability is of great importance for solar cells in space applications as these cells are exposed to hypervelocity impact from particles resulting in extremely large stresses and strains at high strain rates. Particles with diameters in the range of tens to hundreds of microns can penetrate solar cells of spacecrafts and can lead to short circuits and subsequently to a degradation of the power supply. Even though a certain impactor may not be in direct contact with the solar cell, the impact itself creates a compressive stress wave followed by a tensile stress wave. Understanding the failure mechanisms caused by these large stresses and strains is therefore of practical importance. Molecular dynamics (MD) simulations are among the main families of simulation techniques utilized to shed light on mechanical failure of devices resulting in degradation of performance or complete device failure. By using MD simulations, we are able to investigate different physical properties of materials. One efficient way to characterize the predicted structural properties of the materials involved and how they change as a result of mechanical failure is to calculate selective pair distribution functions in the ensemble of calculations.

After a brief review of silicon/silicon nitride usage in solar cell technology, examples of successful applications of pair distribution functions to characterize structural properties of crystalline materials are discussed.

Silicon nitride $\left(\mathrm{Si}_{3} \mathrm{~N}_{4}\right)$ ceramics have been used in a great variety of applications due to their outstanding mechanical properties, especially for high temperature applications. An efficient silicon layer requires a high crystalline quality of the layer, with large crystals and perfect surface passivation. So, the epitaxy temperature must be above $1000{ }^{\circ} \mathrm{C}$. Only ceramic substrates are suitable to withstand temperatures over $1000^{\circ} \mathrm{C}$ [Stollwerck et al., 2001]. Silicon nitride ceramics meet these demands easily. 
Silicon nitride is also well suited as antireflection coatings for silicon solar cells because its optical properties, such as refractive index and absorption coefficient, can be tailored during deposition to match those of silicon solar cells. A good example is the low-temperature remoteplasma PECVD (plasma-enhanced chemical vapor deposition) of $\mathrm{Si}_{3} \mathrm{~N}_{4}$ films [Aberle and Hezel, 1996]. Significant progress in the area of low-temperature passivated silicon solar cells is reported using silicon nitride films fabricated at $300-400^{\circ} \mathrm{C}$ in a remote PECVD system. Due to the low deposition temperatures $\left(\sim 375^{\circ} \mathrm{C}\right)$ and the high refraction index $(\sim 2.2)$, these $\mathrm{Si}_{3} \mathrm{~N}_{4}$ films act as highly efficient surface-passivating antireflection coatings. Compared to thermal oxides grown at high temperature, low-temperature remote-plasma PECVD $\mathrm{Si}_{3} \mathrm{~N}_{4}$ films provide about equal surface passivation on phosphorous-diffused silicon surfaces and significantly superior surface passivation on low-resistivity p-Si wafers. A schematic of such a device is shown in figure 1.

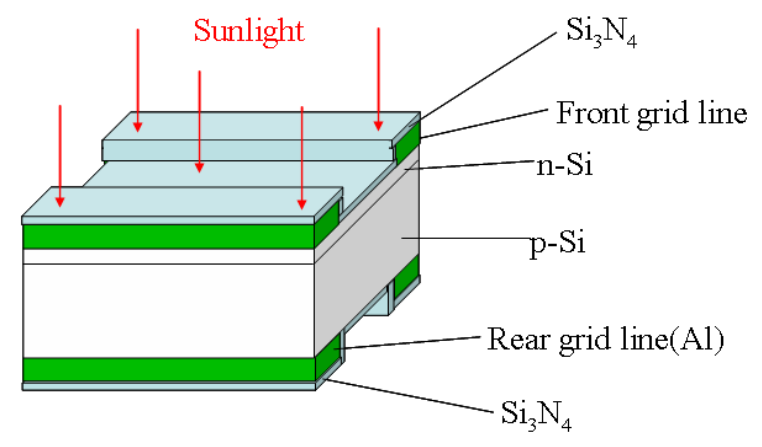

Figure 1: Low-temperature passivated bifacial p-n junction Si solar cell system. Schematics taken from Aberle and Hezel, 1996.

Since silicon nitride films are becoming more technologically important, a detailed understanding of the mechanical response and reliability of these thin structures is becoming very important in their respective applications. Using classical molecular dynamics (MD) simulations, we can explicitly account for both thermal effects and effects arising from the bonding at the interface at the atomistic level.

Pair distribution functions (PDF) are useful to analyze short-range order in different materials [Toby and Egami, 1992]. Traditionally, the PDF is widely used to investigate liquids, glasses, and amorphous materials, but more recently it has been applied to study the structure of crystalline 
materials, too. For example, the crystal structure of sapphire $\left(\alpha-\mathrm{Al}_{2} \mathrm{O}_{3}\right)$ calculated by molecular dynamics simulation was compared with experimental $x$-ray studies at different temperatures [Rambaut et al., 1998]. Interest was focused on the selected interatomic distances (see figure 2) as well as bond angles. The simulation results agreed well with the $x$-ray experimental observations, particularly at $300 \mathrm{~K}$. The calculated interatomic distances shifted from those obtained by $x$-ray experiment by a maximum deviation of only $2 \%$ at $300 \mathrm{~K}$. Moreover, the bond angles exhibited a $5 \%$ average deviation at $300 \mathrm{~K}$. At $2170 \mathrm{~K}$, greater differences between simulation and experiment have been observed. These deviations are due to the empirical nature of the potential, as the different parameters of the potential and the shell model were obtained from fitting of experimental data in equilibrium condition.

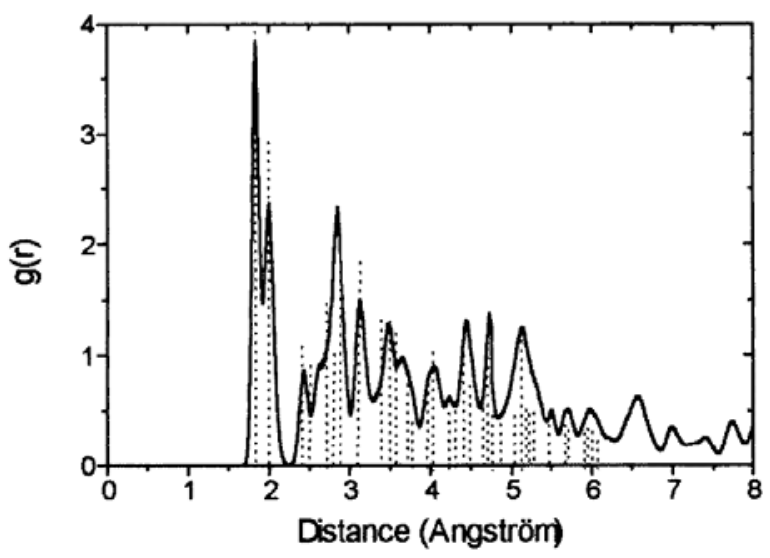

Figure 2: Total radial distribution function of sapphire obtained from simulation (solid line) and from the $x$-ray diffraction studies (dotted line). Taken from Rambaut et al., 1998.

Additionally, pair distribution functions have been used to investigate structural properties (i) in fixed spatial regions as in the case of shock wave structure in Lennard-Jones crystal [Zhakhovskii et al., 1999] and (ii) for earlier specified atoms as in the case of atoms in the interior of nanoclusters and in interfacial regions [Kalia et al., 1997]. In situation (i) the atoms used to calculate the pair distribution function are those atoms whose positions happen to be in a fixed spatial region. In the course of the simulation this region does not change and therefore different atoms may be part of the calculations at one point in time and another and other atoms might have moved to an adjacent spatial region. The other approach (ii) tags atoms at the beginning of the simulation as interior or 
interfacial atoms and uses the interior atoms only to calculate one pair distribution function and the interfacial atoms only to calculate another pair distribution function. Whereas the interior of the nanoparticle may still be considered as bulk like, the interfacial atoms are in a thin-film arrangement of irregular shape and thickness.

The following figure 3 demonstrates a PDF analysis in a quantitative study of shock wave structure in Lennard-Jones crystal [Zhakhovskii et al., 1999], where the unsymmetrical pair distribution function $\rho^{[2]}(r ; z)$, dependent on the distance $r$ in the $x y$ plane between two particles, is calculated in different bins normal to the $z$ axis. As can be seen from the figure, a new first neighbor peak at $r \approx 0.86$ begins to grow (along with the broadening of other peaks) before any significant disorder begins to emerge. Such a behavior is totally different from that observed for a shock wave in a liquid. It may be explained by elastical compression of the lattice before relief of the shear stress coupled with creation of dislocations in the shock front region. The authors also note that the location of this new first peak is almost not shifted when melting the LJ crystal, and that the shift of the first neighbor shell under strong shock compression exceeds those of other shells.

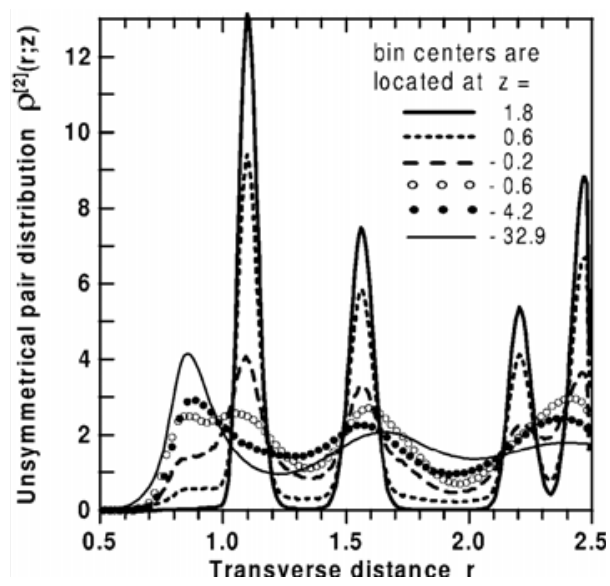

Figure 3: Evolution of the unsymmetrical pair distribution $\rho^{[2]}(r ; z)$ across the shock layer. Taken from Zhakhovskii et al., 1999.

PDF analysis is also performed to investigate in detail the structure of interfacial regions in nanophase $\mathrm{Si}_{3} \mathrm{~N}_{4}$ [Kalia et al., 1997a]. Figure 4 shows $\mathrm{Si}-\mathrm{N}$ pair distribution function for particles 
inside the nanoclusters $g_{S i-N}^{(1)}$ and also for particles in interfacial regions $g_{S i-N}^{(2)}$. The sharp peaks in $g_{S i-N}^{(1)}$ (dashed line) reflect the crystalline structure inside the nanoclusters. In interfacial regions, only the first peak in the pair distribution function $g_{S i-N}^{(2)}$ (solid line) is sharp; the second and third peaks are much broader than those in $g_{S i-N}^{(1)}$. The inset in Figure 4 a) shows that the height of the first peak in $g_{S i-N}^{(2)}$ is 4 times smaller than the height of the first peak in $g_{S i-N}^{(1)}$ and that its position is shifted to a lower value relative to the position of the first peak in $g_{S i-N}^{(1)}$. Along with this shift, there is a decrease in the nearest-neighbor coordination of $\mathrm{Si}$ atoms in interfacial regions.
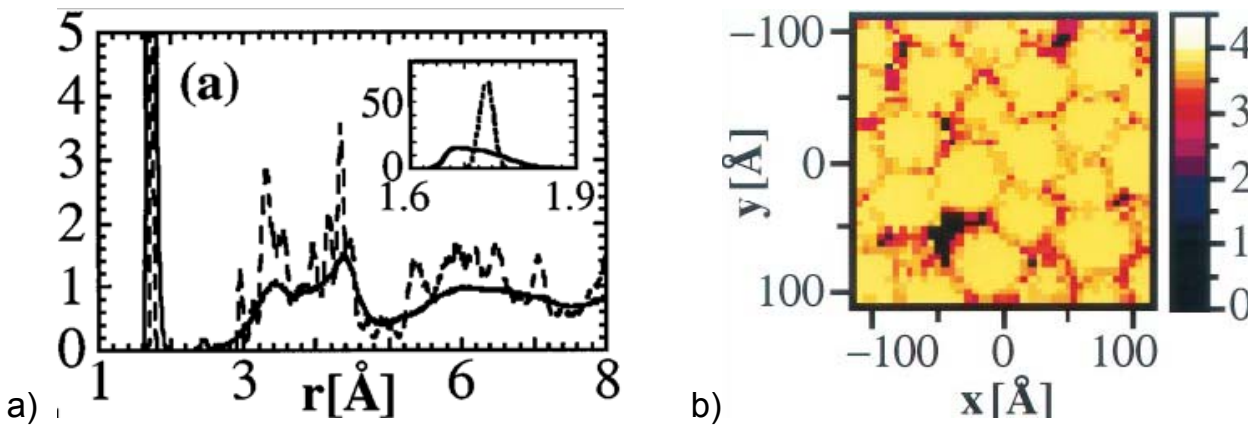

Figure 4: a) Si-N pair-distribution functions in the interior of nanoclusters (dashed lines) and in interfacial regions (solid lines) of the nanophase system. The inset figure shows the first peaks in the pair-distribution functions. b) Spatial distribution of the average Si coordination, projected onto the $x-y$ plane. Taken from Kalia et al., 1997a.

In this thesis, we examine structural properties of specific silicon/silicon nitride interface systems through pair distribution functions. Atomic configurations from MD simulations are used to calculate instantaneous pair distribution functions in bulk silicon, bulk silicon nitride, and silicon/silicon nitride interface. For the interface pair distribution functions we adopt the approach (ii) and use, e.g., interface Si atoms in silicon only to calculate a selective pair distribution function. We have to keep in mind that during the course of the simulation the volume, within which the atoms are, changes its shape (see figure $4 \mathrm{~b}$ ).) 
CHAPTER 1: METHOD: MOLECULAR DYNAMICS (MD) SIMULATION

Over the past few years, with the increase in computer technology and the development of advanced inter-atomic potential functions, molecular dynamics (MD) simulations have played a very important role in physics and related research area. The MD method is an explicit and suitable method for solving classical many-body-system problems. In a molecular dynamics simulation, the time dependent behavior of the molecular system is obtained by integrating Newton's equations of motion using one of the numerical integrators and the potential energy function. The result of the simulation is a time series of configurations; this is called a trajectory or the path followed by each atom in accordance with Newton's laws of motion, from which a number of physical properties, such as thermodynamical and mechanical properties, can be quantitatively analyzed.

When carrying out an MD simulation, coordinates, velocities, and particle identity of the atoms in the system are saved; these are then used for the analysis. Time dependent properties can be displayed graphically, where one of the axes corresponds to time and the other to the quantity of interest, such as energy, temperature, stress, etc. Average properties can be calculated and compared to experimental values. Molecular dynamics simulations can also be visualized to understand conformational changes at an atomic level when combined with molecular graphics programs which can display the atomic properties of interest.

MD simulations of complex phenomena require large system size and long simulation times such as $10^{5}-10^{7}$ particles and $10^{4}-10^{6}$ time steps [Vashishta et al., 1994]. So an application to complex systems requires the use of large scale parallel computer architectures. Recently, with the dramatic development of computer technology, MD simulations based on parallel computations offer a specific way to build up a bridge between theory and experiment. We may test a theory by conducting simulations using the same model, or carry out simulations on the computer that are difficult or impossible in the laboratory.

Each and every atom follows Newton's second law may be written as [Allen and Tildesley, 1987; Allen, 2004] 


$$
m_{i} \ddot{\vec{r}}_{i}=\vec{f}_{i}, \vec{f}_{i}=-\frac{\partial}{\partial \vec{r}_{i}} U
$$

where $\vec{f}_{i}$ is the force acting on atom $i$, and it is usually derived from a potential energy $U\left(\vec{r}^{N}\right)$, where $\vec{r}^{N}=\left(\vec{r}_{1}, \vec{r}_{2} \ldots . . \vec{r}_{N}\right)$ is the complete set of $3 N$ atomic coordinates.

The potential energy may be divided into terms depending on the coordinates of individual atoms, pairs, triplets etc:

$$
U\left(\vec{r}^{N}\right)=\sum_{i} u\left(\vec{r}_{i}\right)+\sum_{i} \sum_{i<j} v\left(\vec{r}_{i}, \vec{r}_{j}\right)+\ldots
$$

The first term of equation (1.2) is the effect of an external field on the system. The second term is the pair potential. We usually concentrate on this term and three-body terms and neglect higher order interactions.

One of the most widely used MD algorithms for solving Newton's equations is the Verlet algorithm. The method is based on positions $\vec{r}(t)$, accelerations $\vec{a}(t)$, and the positions $\vec{r}(t-\delta t)$ from the previous step.

$$
\vec{r}(t+\delta t)=2 \vec{r}(t)-\vec{r}(t-\delta t)+\delta t^{2} \vec{a}(t)
$$

Using Taylor expansion about $r(t)$ :

$$
\begin{aligned}
& \vec{r}(t+\delta t)=\vec{r}(t)+\delta t \vec{v}(t)+(1 / 2) \delta t^{2} \vec{a}(t)+\ldots \ldots \\
& \vec{r}(t-\delta t)=\vec{r}(t)-\delta t \vec{v}(t)+(1 / 2) \delta t^{2} \vec{a}(t)-\ldots \ldots
\end{aligned}
$$

The velocities are not needed to compute the trajectories, but they are useful for estimating the kinetic energy. They may be obtained from the formula:

$$
\vec{v}(t)=\frac{\vec{r}(t+\delta t)-\vec{r}(t-\delta t)}{2 \delta t}
$$

There are various, essentially equivalent versions of the Verlet algorithm. Here we use on the velocity Verlet algorithm, which may be written as 


$$
\begin{aligned}
& \vec{r}(t+\delta t)=\vec{r}(t)+\delta t \vec{v}(t)+(1 / 2) \delta t^{2} \vec{a}(t) \\
& \vec{v}(t+\delta t)=\vec{v}(t)+\frac{1}{2} \delta t[\vec{a}(t)+\vec{a}(t+\delta t)]
\end{aligned}
$$


CHAPTER 2: SETUP OF SIMULATION AND MODEL OF INTERFACE

Silicon is an extensively studied material due to its wide range of applications. Several interaction potentials between $\mathrm{Si}$ atoms within silicon crystals have been developed and used in a variety of applications including Stillinger and Weber 1985, Tersoff 1986, Chelikowsky 1988, Cook and Clancy 1993, Juan et al 1996, Justo et al 1998, Choudhary and Clancy 2005, Demkowicz and Argon 2005, 2005a and Rosselt et al 2005. We use the Stillinger-Weber potential to describe the interactions in bulk silicon. (see Eq. 2.1)

$$
v\left(r_{i j}\right)=C_{1}\left(\left(\frac{\sigma^{*}}{r_{i j}}\right)^{n_{i j}}-C_{2}\right) \exp \left(\frac{\sigma}{r-\sigma_{a}}\right)
$$

where

$n_{i j}=4, \mathrm{C}_{1}=.14741 \times 10^{-19} \mathrm{~J}, C_{2}=.24478 \times 10^{-19} \mathrm{~J}, \sigma=2.095 \AA, \sigma_{\mathrm{a}}=3.7712 \AA, \sigma^{*}=2.1370 \AA$

For silicon nitride an interaction model including two-body and three-body terms [Vashishta et al., 1996] has been implemented. The two-body terms account for steric repulsion, screened Coulomb interaction, and charge-dipole interactions. The three-body terms ensure that ideal crystalline bond angles have minimum energy. (see Eq. 2.2-2.6)

Two-body terms for Si-N, Si-Si, and N-N interactions are:

$$
\begin{gathered}
v\left(r_{i j}\right)=v_{i j}^{(1)}\left(r_{i j}\right)+v_{i j}^{(2)}\left(r_{i j}\right)+v_{i j}^{(3)}\left(r_{i j}\right) \quad(2.2) \\
v_{i j}^{(1)}\left(r_{i j}\right)=A_{i j}\left(\frac{\sigma_{i j}}{r_{i j}}\right)^{n_{i j}} \quad \text { Steric Repulsion } \quad(2.3) \\
v_{i j}^{(2)}\left(r_{i j}\right)=B_{i j}\left(\frac{Z_{i} Z_{j}}{r_{i j}}\right) \exp \left(-r_{i j} / r_{0}\right) \quad \text { Coulomb } \quad(2.4) \\
v_{i j}^{(3)}\left(r_{i j}\right)=B_{i j} \frac{\left(\alpha_{i} Z_{j}^{2}+\alpha_{j} Z_{i}^{2}\right) / 2}{r_{i j}^{4}} \exp \left(-r_{i j} / r_{4}\right) \quad \text { Charge-dipole }
\end{gathered}
$$


Three-body terms for Si-N-Si and N-Si-N configurations are:

$$
w_{i j k}\left(r_{i j}, r_{i k}\right)=B_{i j k}\left(\cos \theta_{i j k}-\cos \theta_{0}\right)^{2}
$$

where

$$
\begin{aligned}
& A_{i j}=2 \times 10^{-19} \mathrm{~J}, B_{i j}=23.07 \times 10^{-19} \mathrm{~J}, r_{0}=2.5 \AA, r_{4}=2.5 \AA, \sigma_{i j}=\sigma_{i}+\sigma_{i}, \sigma_{i}^{S i}=0.47 \AA, \\
& \sigma_{i}^{N}=1.3 \AA, Z_{i}^{S i}=1.472 \mathrm{e}, Z_{i}^{N}=-1.104 \mathrm{e}, \alpha_{i}^{S i}=0.0 \AA^{3}, \alpha_{i}^{N}=3.0 \AA^{3}, n_{i j}^{S i-S i}=11, n_{i j}^{S i-N}=9, n_{i j}^{N-N}=7, \\
& \text { and } B_{i j k}^{S i-N-S i}=20 \times 10^{-19} \mathrm{~J}, B_{i j k}^{N-S i-N}=10 \times 10^{-19} \mathrm{~J}, \theta_{0}^{S i-N-S i}=120, \theta_{0}^{N-S i-N}=109.47 .
\end{aligned}
$$

This model for silicon nitride has been applied successfully to calculate structural, mechanical, and dynamical properties of crystalline, amorphous, and nanophase silicon nitride [Loong et al., 1995; Vashishta et al., 1995, Nakano et al., 1995; Vashishta et al., 1996a; Kalia et al., 1997; Tsuruta et al., 1998.]

Our model for the silicon/silicon nitride interface [Bachlechner et al., 1999] distinguishes between $\mathrm{Si}$ atoms in the silicon crystal and $\mathrm{Si}$ and $\mathrm{N}$ atoms in silicon nitride. In addition, the atoms at or near the interface have different charge transfer and therefore have to be treated differently. Based on LCAO (Linear Combination of Atomic Orbitals) electronic structure calculations [Zhao and Bachlechner, 1997; 1998] for the crystalline silicon/silicon nitride interface, this system may be adequately modeled as an eight-component system (see table 1 and figure 5). Two-by-two unit cells of $\mathrm{Si}(111)$ correspond to one unit cell of $\mathrm{Si}_{3} \mathrm{~N}_{4}(0001)$ in the respective interface planes with a small lattice mismatch of $1.12 \%$. We model silicon with a larger lattice constant so that it perfectly matches silicon nitride. This corresponds to changing the parameter $\sigma^{*}=0.20951 \mathrm{~nm}$ in the original Stillinger-Weber potential [Stillinger and Weber, 1985] to $\sigma^{*}=0.21370 \mathrm{~nm}$ in the simulations presented here.

$\mathrm{Si}$ atoms in the top layer of silicon form bonds to $\mathrm{N}$ atoms in the bottom layer of silicon nitride leaving dangling bonds of $\mathrm{Si}$ atoms in the interface layer of silicon nitride. The assumptions for the interface model include that the interface is sharp which Kim and Yeom (2003) justify experimentally. Additionally, we assume that the bond lengths and the bond angles at and across the interface are comparable to those in bulk silicon nitride. 

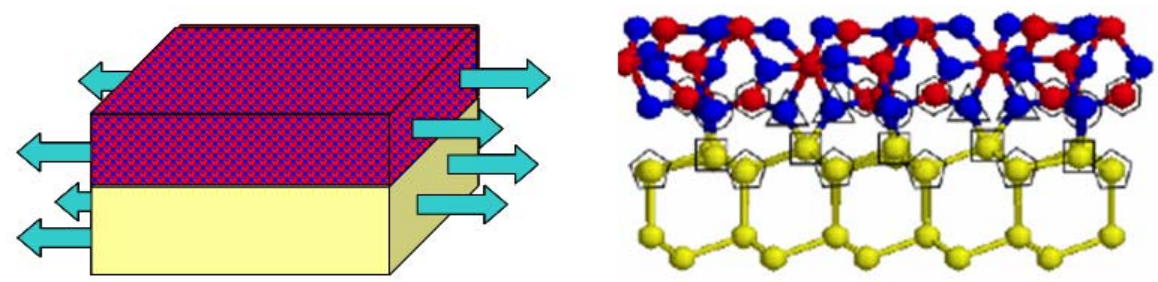

Figure 5: System structure of $\mathrm{Si}(111) / \mathrm{Si}_{3} \mathrm{~N}_{4}(0001)$ interface. Yellow spheres represent silicon, red and blue spheres represent silicon nitride. In silicon at the interface, pentagons denote $\mathrm{Si}$ atoms of type 7 and squares denote $\mathrm{Si}$ atoms of type 8 . In silicon nitride at the interface, circles denote $\mathrm{N}$ atoms of type 4, triangles denote $\mathrm{N}$ atoms of type 5, and hexagons denote Si atoms of type 2.

Figure 5 shows the schematics and atomic positions in the $\mathrm{Si}(111) / \mathrm{Si}_{3} \mathrm{~N}_{4}(0001)$ interface. Within the silicon interface double layer every $\mathrm{Si}$ atom of type 7 is bonded to three $\mathrm{Si}$ atoms of type 8 and one $\mathrm{Si}$ atom of type 6 in the bulk of silicon. Every $\mathrm{Si}$ atom of type 8 is bonded to three $\mathrm{Si}$ atoms of type 7 and one $\mathrm{N}$ atom of type 4 or 5 in the interface layer of silicon nitride.

Our simulations use a $\mathrm{Si}_{3} \mathrm{~N}_{4}$ film of height $44.49 \AA$ and a Si substrate of height $95.24 \AA$ (see figure 5). The horizontal dimensions of the interface are $269.36 \AA \times 279.94 \AA$. The total atom number in both silicon and silicon nitride is 668,160. More detailed descriptions are presented in table 1. 


\begin{tabular}{|l|l|l|l|}
\hline Atom ID & Atom Type & Atom Description & Atom Number \\
\hline 1 & $\mathrm{Si}^{4+}$ & silicon in bulk silicon nitride & 133920 \\
\hline 2 & $\mathrm{Si}^{3+}$ & silicon in silicon nitride at the interface & 4320 \\
\hline 3 & $\mathrm{~N}^{3-}$ & nitrogen in bulk silicon nitride & 178560 \\
\hline 4 & $\mathrm{~N}^{2-}$ & $\begin{array}{l}\text { nitrogen in silicon nitride at the interface (fully } \\
\text { coordinated within the interface plane) }\end{array}$ & 1440 \\
\hline 5 & $\mathrm{~N}^{3-}$ & $\begin{array}{l}\text { nitrogen in silicon nitride at the interface (one } \\
\text { dangling bond) }\end{array}$ & 4320 \\
\hline 6 & $\mathrm{Si}$ & $\begin{array}{l}\text { silicon in bulk silicon } \\
\text { silicon in silicon at the interface (fully } \\
\text { coordinated) }\end{array}$ & 5760 \\
\hline 7 & $\mathrm{Si}$ & $\begin{array}{l}\text { silicon in silicon at the interface (one dangling } \\
\text { bond) }\end{array}$ & 5760 \\
\hline 8 & $\mathrm{Si}$ & 334080 \\
\hline
\end{tabular}

Table 1: Detailed MD simulation system description 


\section{CHAPTER 3: PAIR DISTRIBUTION FUNCTIONS}

\section{Theoretical Background}

The structure of the crystalline silicon/silicon nitride interface may be characterized by pair distribution functions for the atomic positions. This function gives the probability of finding a pair of atoms a distance $r$ apart, relative to the probability expected for a completely random distribution at the same density. In the canonical ensemble, the pair distribution function can be written as follows [Allen and Tildesley, 1987] :

$$
g\left(\vec{r}_{1}, \vec{r}_{2}\right)=\frac{N(N-1)}{\rho^{2} Z_{N V T}} \int d \vec{r}_{3} d \vec{r}_{4} \ldots . . d \vec{r}_{N} \exp \left(-\beta U\left(\vec{r}_{1}, \vec{r}_{2}, \ldots . . \vec{r}_{N}\right)\right)
$$

In Eq.(3.1), $N$ is number of atoms, $\rho=N / V$ is density of atoms, $V$ is the sample volume before applying external strains, $Z_{N V T}$ is partition function, $U$ is potential energy, and $\beta=1 / K_{B} T$. Obviously the choice $i=1, j=2$ is arbitrary in a system of identical atoms. An equivalent definition takes an ensemble average over pairs [Allen and Tildesley, 1987; Vesely, 2001]:

$$
\begin{aligned}
g(r) & =\rho^{-2}<\sum_{i} \sum_{j \neq i} \delta^{3}\left(\vec{r}_{i}\right) \delta^{3}\left(\vec{r}_{j}-\vec{r}\right)> \\
& =\frac{V}{N^{2}}<\sum_{i} \sum_{j \neq i} \delta^{3}\left(\vec{r}-\vec{r}_{j i}\right)> \\
& =\frac{V}{4 \pi r^{2} N^{2}}<\sum_{i} \sum_{j \neq i} \delta\left(r-r_{j i}\right)>\quad \text {, with } \vec{r}_{j i}=\vec{r}_{j}-\vec{r}_{i} \text {, i.e., } r_{j i}=\left|\vec{r}_{j}-\vec{r}_{i}\right| .
\end{aligned}
$$

We stress however that Eq. (3.2) applies strictly only to fluids which are, by definition, spatially uniform and isotropic state of matter. Due to this, fluids exhibit translationally and rotationally invariant correlation functions. Thus, in particular, in liquids, the pair distribution function in Eq. (3.1) depends only on the magnitude of the difference between $\vec{r}_{1}$ and $\vec{r}_{2}$. Crystalline solids however break both the rotational and translational symmetries, and the pair distribution function in Eq. (3.1) is not a function of the magnitude of the difference between $\vec{r}_{1}$ and $\vec{r}_{2}$. Due to this, for crystals, the three various entries in Eq. (3.2) are generally not equal to each other. Still, the last term in (3.2), 
representing the translationally and rotationally averaged pair distribution function, can be used as a theoretical probe of various interesting structural properties of crystalline solids. For briefness, in the following discussions we call this last term of Equation (3.2) as the pair distribution function $g(r)$. We calculate it here from numerical simulations of atomic dynamics.

For the results presented in the following, we use Langevin dynamics [Abraham and Gao, 2000] to hold the temperatures of the interfaces at $300 \mathrm{~K}, 600 \mathrm{~K}$, and $900 \mathrm{~K}$, respectively. In our studies here, we compute the non-equilibrium equal-time pair distribution functions which, in contrast to their equilibrium counterpart Eq. (3.1), do depend on time. We calculate this quantity as the instantaneous value of the sum in the last term in Eq. (3.2). We apply strain parallel to the interface in $x$-direction (see figure 5) to investigate the tensile strength of the silicon/silicon nitride interface. Periodic boundary conditions are used in the $y$-direction. A 5- $\AA$-thick stripe of atoms on the left and on the right in figure 5 is held and pulled to the left and to the right, respectively. Pulling $1 \%$ within 2,000 time steps using a time step of 6 fs corresponds to a strain rate of $0.0025 \mathrm{ps}^{-1}=2.5^{\star} 10^{9} \mathrm{~s}^{-1}$.

\section{Pair distribution functions in bulk silicon}

Silicon crystals have diamond structure with tetrahedral bonding (see figure 6). Four bonding neighbors that sit at the corners of a tetrahedron surround each silicon atom; the bond angle is therefore $109.47^{\circ}$. The first nearest neighbor distance or bond length is $2.38 \AA$ for modified silicon model in which $\mathrm{Si}(111)$ perfectly lattice matches $\mathrm{Si}_{3} \mathrm{~N}_{4}(0001)$. Geometric values of further nearest neighbor distances are calculated from ideal first nearest neighbor distances and ideal bond angles using the law of cosine. For the second nearest neighbor distance a value of $3.89 \AA$ is obtained. Each of the four bonding neighbors is bonded to three additional neighbors resulting in a total of twelve second-nearest neighbors. 

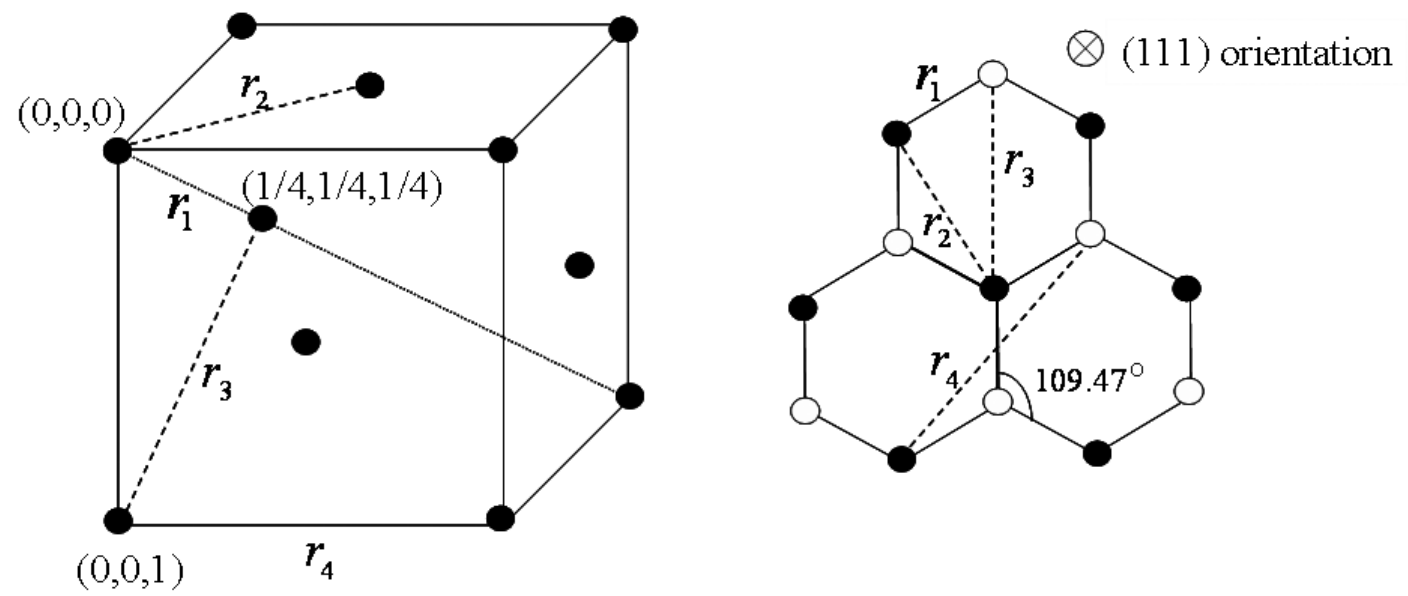

Figure 6: Atomic positions for bulk silicon in its diamond structure. (a) Conventional unit cell. Only the atoms on the top, right side, and front faces are displayed as well as the atom along the cube diagonal. (b) View onto a (111) plane.

The pair distribution function of $\mathrm{Si}$ atoms in the bulk silicon crystal (see figure 7), i.e. $g(6,6)$, reflects the diamond structure as discussed above. At higher temperatures, the atoms experience larger random fluctuations about their crystalline positions. As a consequence, the bond lengths as well as the distance to next and further nearest neighbors increase or decrease resulting in a broader distribution about the ideal distances with a smaller height (see figure 7.) For example, the second and third nearest neighbor peaks are well separated at $300 \mathrm{~K}$, whereas they overlap at $600 \mathrm{~K}$ and $900 \mathrm{~K}$. This effect is even more pronounced at further nearest neighbors where peaks almost disappear and become shoulders in adjacent peaks. The peak position, however, does not change with increasing temperature. This is consistent with thermal expansion coefficient of silicon which results in changes of nearest neighbor distances that are below the resolution accessible in the simulation. Table 2 shows the computational values of several nearest neighbor distances extracted from figure 7 in comparison with the geometrical values of them. 

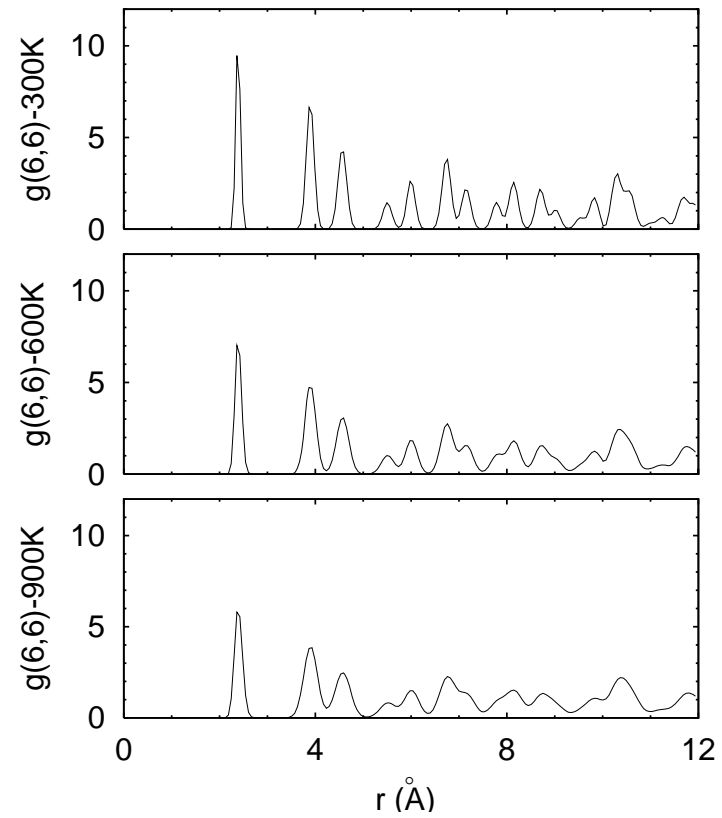

Figure 7: Si-Si pair distribution function $g(6,6)$ in bulk silicon at different temperatures

\begin{tabular}{|l|l|l|}
\hline Si-Si in bulk silicon $(300 K)$ & Geometrical $(\AA)$ & Computational $(\AA)$ \\
\hline $1^{\text {st }}$ nearest-neighbor distance & $2.38(4$ nearest-neighbor $)$ & 2.39 \\
\hline $2^{\text {nd }}$ & $3.89(12$ nearest-neighbor $)$ & 3.89 \\
\hline $3^{\text {rd }}$ & $4.55(9$ nearest-neighbor $)$ & 4.58 \\
\hline $4^{\text {th }}$ & $5.50(6$ nearest-neighbor $)$ & 5.51 \\
\hline
\end{tabular}

Table 2: Comparison of geometrical and computational values of the first 4 nearest neighbor distances in bulk silicon at $300 \mathrm{~K}$. Geometric values are calculated from ideal $1^{\text {st }}$ nearest neighbor distances and ideal bond angles using law of cosine.

\section{Pair distribution functions in bulk silicon nitride}

Silicon nitride has a hexagonal crystal lattice structure. The ideal bond length of every Si-N pair is $1.73 \AA$. This is also the first nearest neighbor distance. Every $\mathrm{N}$ atom is bonded to three $\mathrm{Si}$ atoms. The bond angle with a $\mathrm{N}$ atom in the center is $120^{\circ}$. Every $\mathrm{Si}$ atom is bonded to four $\mathrm{N}$ atoms in a tetrahedral configuration. The bond angle with a Si atom in the center is therefore $109.47^{\circ}$. Further 
nearest neighbor distances can be geometrically calculated using the above information. Selected distances are displayed in figure 8 , and table 3 lists the properties up to the tenth nearest neighbors.

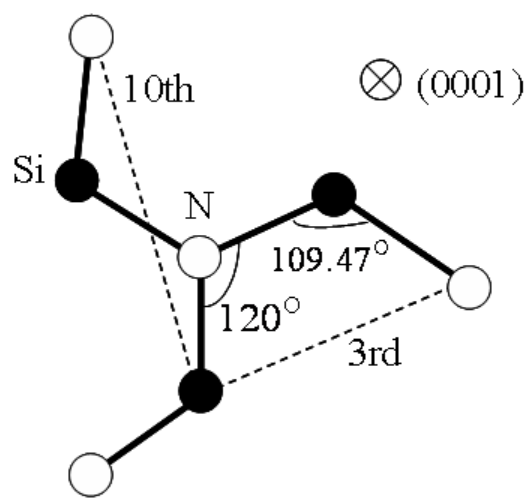

Figure 8: Sample for geometrical calculation of nearest neighbor distances within a $\mathrm{Si}_{3} \mathrm{~N}_{4}$ unit. According to this figure the $3^{\text {rd }}$ and $10^{\text {th }}$ nearest neighbor distances can be calculated. Similar approach is applied for other nearest neighbors.

\begin{tabular}{|l|l|l|}
\hline Si-N in bulk silicon nitride & Geometrical $(\AA)$ & Computational $(\AA)$ \\
\hline $1^{\text {st }}$ nearest neighbor distance & 1.73 & 1.73 \\
\hline 2nd & 2.94 & 2.96 \\
\hline 3rd & 3.13 & 3.17 \\
\hline 4 th & 3.31 & 3.32 \\
\hline 5 th & 3.53 & 3.53 \\
\hline 6 th & 3.78 & 3.77 \\
\hline 7 th & 3.98 & 3.95 \\
\hline 8th & 4.14 & 4.19 \\
\hline 9th & 4.21 & 4.19 \\
\hline 10 th & 4.35 & 4.34 \\
\hline
\end{tabular}

Table 3: Comparison of geometrical and computational values of the first 10 nearest neighbor distances in silicon nitride pairs. 
Picking Si atoms in bulk silicon nitride, i.e. atom type 1, we calculate distances to $\mathrm{N}$ atoms in bulk silicon nitride only, i.e. atom type 3 , to obtain the pair distribution function denoted as $g(1,3)$. The further nearest neighbors in the Si-N pair distribution function $g(1,3)$ result in multiple overlapping peaks due to the complex structure within silicon nitride. Figure 9 shows the pair distribution functions $g(1,3)$ with figure 9 (b) identifying the respective distances given in table 3 (compare also with dashed lines in figure 4.)
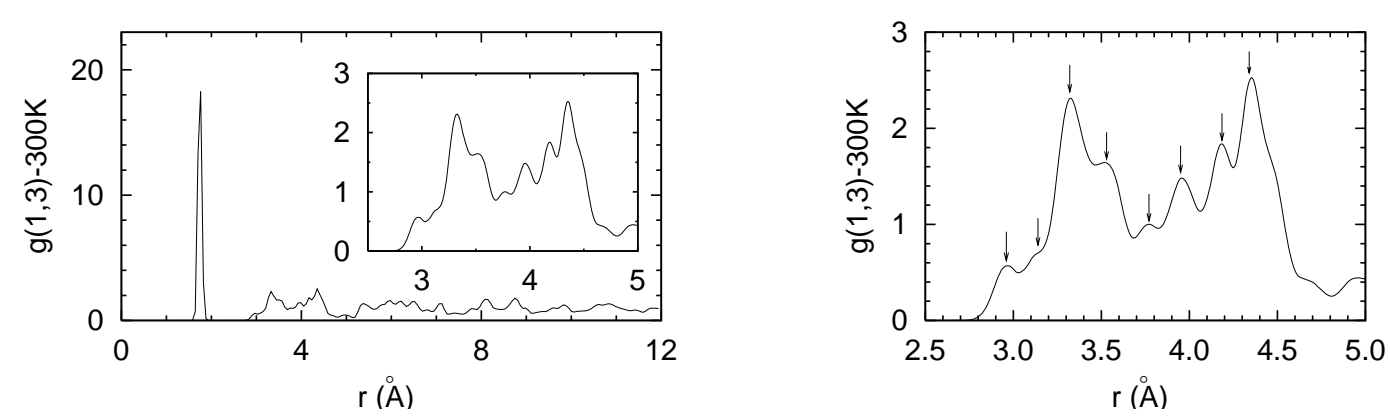

Figure 9: (a) Si-N pair distribution function in bulk silicon nitride at 300K. (b) A magnified picture from (a). The arrows indicate the peak positions (see also table 3.)

Figure 10 shows pair distribution functions and corresponding visualization at specific values of strain. At about $8 \%$ strain, a crack opens at the left side of the film. Until $8 \%$, the first peak in the pair distribution function broadens visibly, its height decreases compared to its value at $1 \%$ strain. After another crack opens at the right side of the film between 8 and $9 \%$ strain, the centerpiece of $\mathrm{Si}_{3} \mathrm{~N}_{4}$ breaks off and returns to an essentially unstretched configuration afterwards. This is reflected in the pair distribution function returning to almost its original shape. 

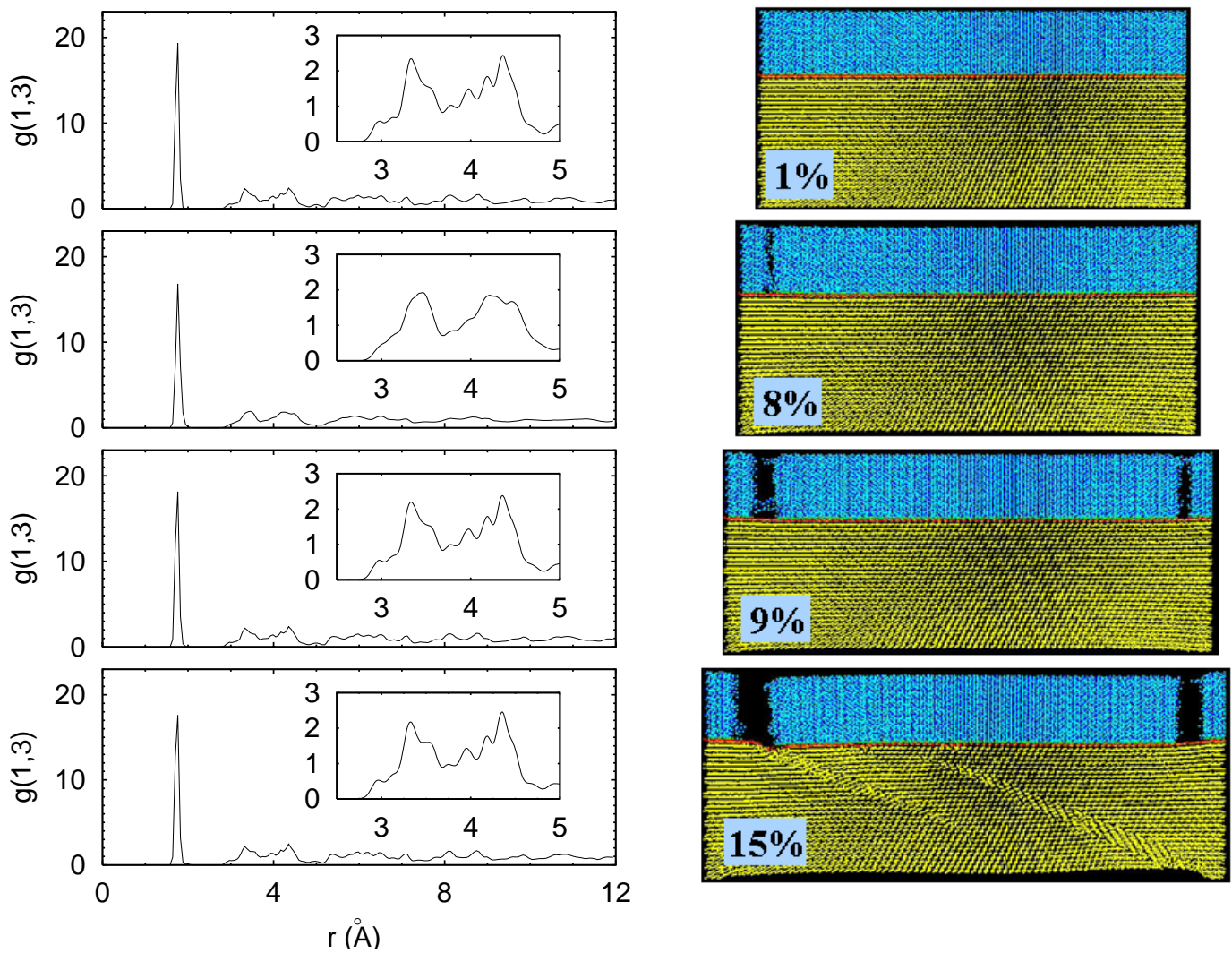

Figure 10: Si-N pair distribution function in bulk silicon nitride under different strains at 300K.

For additional analysis, we extracted the heights of the first peaks in the pair distribution function (as seen in figure 10). Figure 11 shows the heights of the first peaks in pair distribution function versus strains. Because bonds are enlarged in stretching direction whereas shortened in direction perpendicular to stretching direction, the first peak heights decrease until $8 \%$. Fracture occurs around $8 \%$ for all 3 different temperatures. After that, because the center piece is disconnected from the region on the left and the right that is being pulled, it can relax to almost the unstrained configuration which is reflected in the fact that the peak height of the first peak returns almost to its original value. 


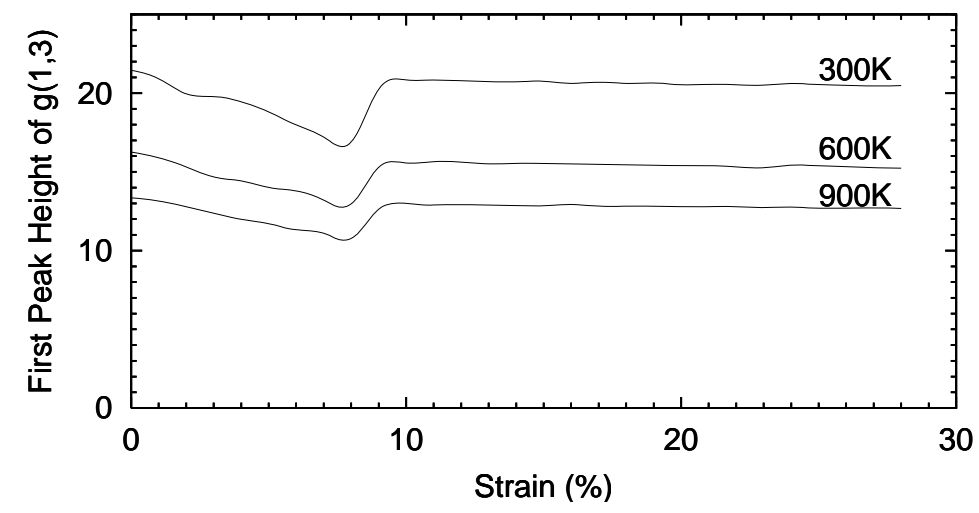

Figure 11: Height of the first peak of $g(1,3)$ in bulk silicon nitride as depicted in figure 10 at different temperatures versus externally applied tensile strain.

\section{Pair distribution functions at the interface}

Of special interest for our analysis are atoms within the silicon double layer at the interface, i.e. atom types 7 and 8. Focusing on nearest neighbor distances amongst Si atoms of type 7 only, we need to note that the first nearest neighbors do not represent bonding neighbors. The distance corresponds to the second-nearest neighbors in bulk silicon as seen in figure 12. Using the first nearest neighbor distance or bond length of $2.38 \AA$ for our modified silicon model and the angle of $109.47^{\circ}$, we can calculate geometrical values of further nearest neighbor distances through the law of cosines.

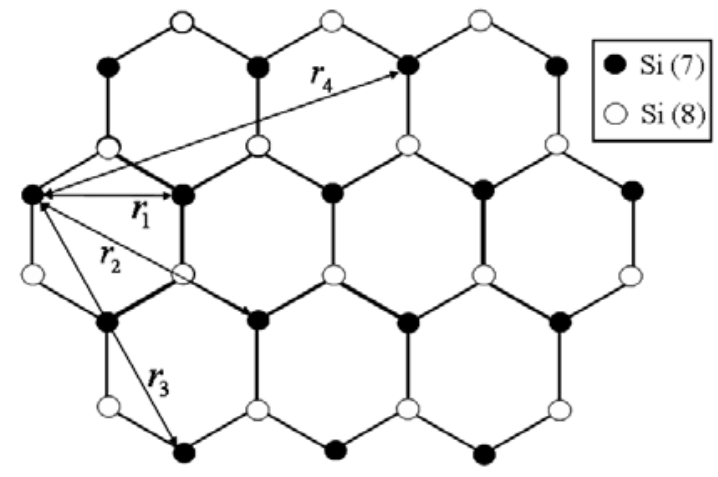

Figure 12: The first 4 nearest neighbor distances in the interface for $\mathrm{Si}(7)-\mathrm{Si}(7)$. 
Two different coherent interface phases leading to a local energy minimum configuration have been considered earlier [Lidorikis et al., 2005]. The phase D1 consists of the $\mathrm{N}$ atoms of type 4 in the interface plane of silicon nitride being positioned directly on top of the Si atoms of type 8 in the interface double layer of silicon (see figure 13.a)) with a bond length of $1.75 \AA$. The phase D2 is obtained by slip of the silicon nitride along silicon (see figure 13.b)) at the interface in a way that the $\mathrm{N}$ atoms of type 4 are positioned in the center of the hexagons formed by the silicon interface double layer. The nitrogen atom is then bonded to three $\mathrm{Si}$ atoms across the interface with a bond length around $2.5 \AA$. It has been found that successive D1 -> D2 and D2 -> D1 slips relax the mismatch strain in form of a triangular superlattice of interfacial domains [Lidorikis et al., 2005]. In the simulation presented here the initial configuration was in phase D1.
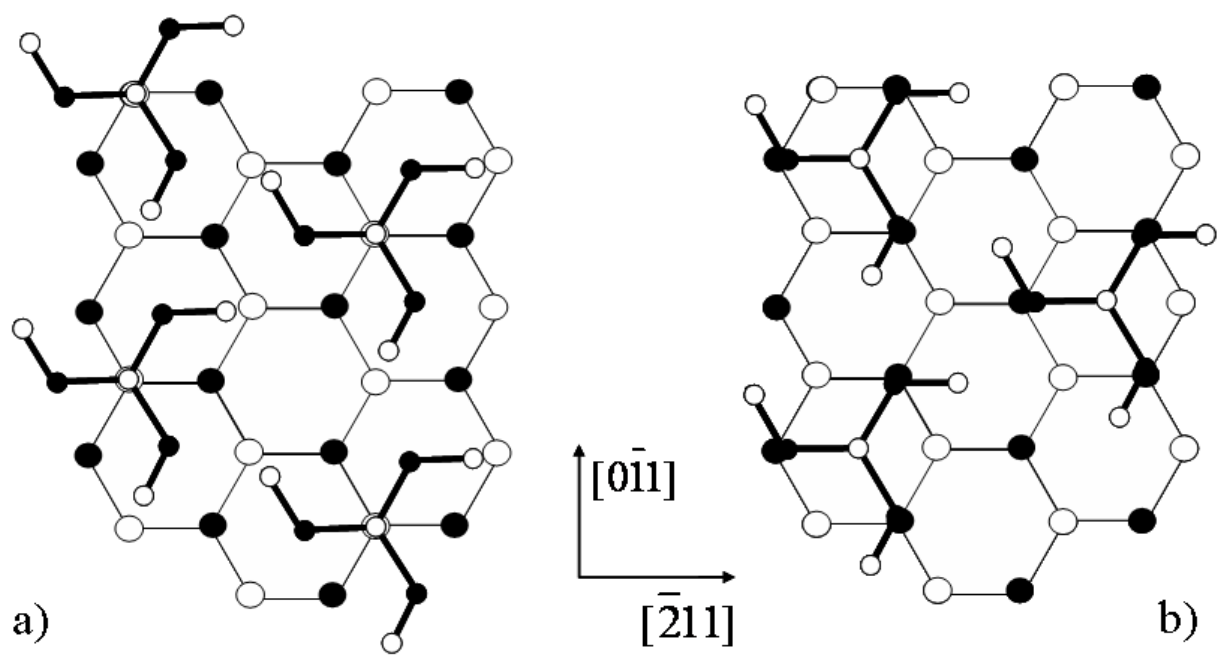

Figure 13: The two different coherent $\mathrm{Si}(111) / \mathrm{Si}_{3} \mathrm{~N}_{4}(0001)$ interface phases. Big circles denote $\mathrm{Si}$ atoms in silicon at the interface and small circles denote $\mathrm{Si}$ and $\mathrm{N}$ atoms in the silicon nitride interface plane. Big open circles are $\mathrm{Si}(8)$ atoms, big full circles are $\mathrm{Si}(7)$ atoms. Small open circles are $\mathrm{N}(4)$ and $\mathrm{N}(5)$ atoms, small full circles are $\mathrm{Si}(2)$ atoms. (a) In phase $\mathrm{D} 1, \mathrm{~N}$ atoms of type 4 in the interface plane of silicon nitride are almost directly on top of Si atoms of type 8 in the interface plane of silicon. (b) In phase D2, the silicon nitride film slipped in a way that $\mathrm{N}$ atoms of type 4 in the interface plane of silicon nitride are almost in the center of the hexagons formed by Si atoms at the interface. 
In figure 14, we give the pair distribution function for $\mathrm{Si}(7)$. These atoms occupy $9.41 \AA$ thick layer (six atomic monolayers) at the top of the silicon substrate. The Si-Si pair distribution functions $g(7,7)$ in figure 14 show that all peaks broaden and become less high as strain is applied. At a strain of $14 \%$, two new features are observed. First, a new peak develops at a distance of $2.35 \AA$, which corresponds to a distance suggesting a bond between two originally unbonded $\mathrm{Si}$ atoms at the interface of type 7 . With increasing strain, more and more distances suggesting a bond between two originally unbonded Si atoms occur. Secondly, there is an additional peak between the original second and third peaks reflecting again possibility of bonding between two Si(7). With increasing strain those peaks overlap.

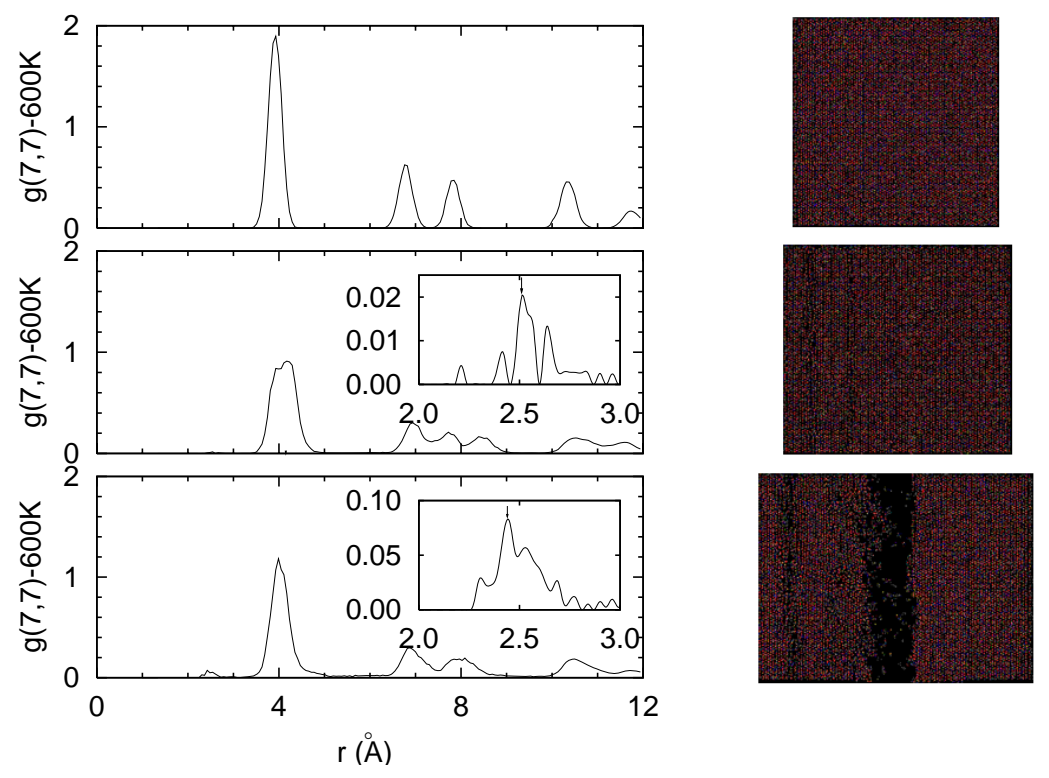

Figure 14: Si-Si pair distribution functions, $g(7,7)$, at the interface at strains of $1 \%, 14 \%$, and $28 \%$, respectively, from top to bottom, and corresponding visualization of the interface silicon double layer. The insets show new peak(s) which correspond the distance suggesting a bond between two originally unbonded $\mathrm{Si}$ atoms at the interface of type 7 before original first peaks. The arrows indicate the chosen peak positions for figure 15.

Similarly, table 4 shows the computational values of several nearest neighbor distances in comparison with the geometrical values of them. 


\begin{tabular}{|l|l|l|}
\hline $\operatorname{Si}(7)-\operatorname{Si}(7)$ in interface $(300 K)$ & Geometrical $(\AA)$ & Computational $(\AA)$ \\
\hline $1^{\text {st }}$ nearest-neighbor distance & 3.89 & 3.89 \\
\hline $2^{\text {nd }}$ nearest-neighbor distance & 6.74 & 6.80 \\
\hline $3^{\text {rd }}$ nearest-neighbor distance & 7.78 & 7.82 \\
\hline $4^{\text {th }}$ nearest-neighbor distance & 10.3 & 10.3 \\
\hline
\end{tabular}

Table 4: Comparison of geometrical and computational values of the first 4 nearest neighbor distances in $\mathrm{Si}(7)-\mathrm{Si}(7)$ in interface silicon. Geometric values are calculated from ideal $1^{\text {st }}$ nearest neighbor distances and ideal bond angles using law of cosine.

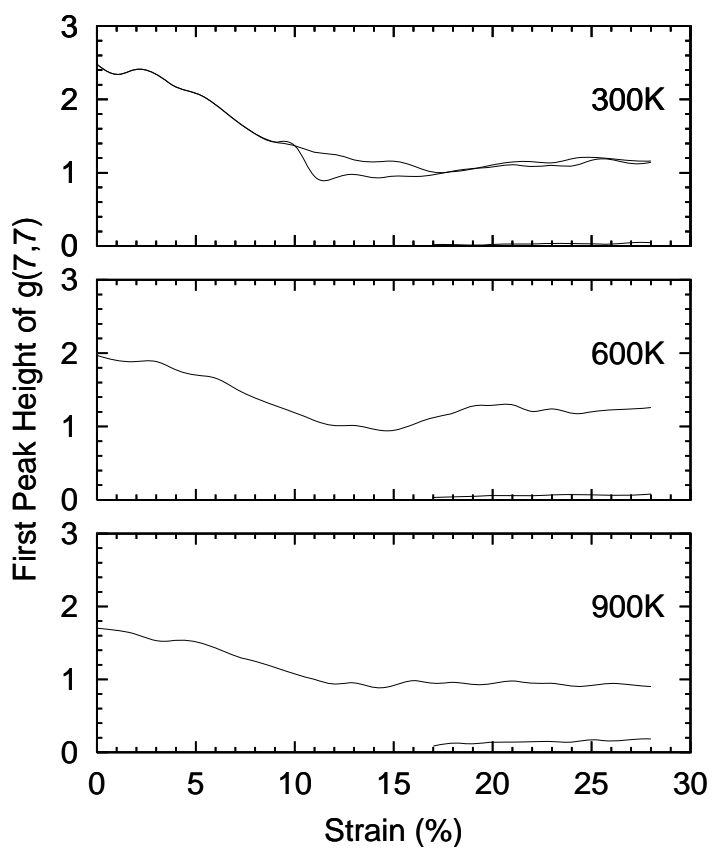

Figure 15: Height of the first peak(s) of $g(7,7)$ at silicon interface versus strain at different temperatures. Main line(s) starting from zero strain denote the original first peak positions. For $300 \mathrm{~K}$, we mark two different first peak positions at each specific strain rate. For $600 \mathrm{~K}$ and $900 \mathrm{~K}$, we choose the highest point at the top of first peak (as depicted in figure 14) as first peak positions. An additional line starts at $17 \%$ for all three temperatures denoting the new peak positions as indicated by arrows in insets in figure 14 . 
Figure 15 shows the heights of the first peaks as they vary with applied strain in $g(7,7)$. At all three temperatures, additional peaks for smaller distances appear at $17 \%$. The heights of the additional peaks increase most prominently for the highest temperature.

As discussed in the beginning of this section, there are two different coherent interface phases corresponding to local energy minimum configurations denoted by D1 and D2. The most prominent difference between these two configuration lies in the bond length between $\mathrm{N}$ atoms of type 4 in the silicon nitride interface layer and $\mathrm{Si}$ atoms of type 8 in the silicon interface double layer. The interface thickness is taken to be $2.96 \AA$ and consists of the top layer in silicon, Si atoms type 8 , and the bottom layer in silicon nitride, Si atoms type 2 and nitrogen atoms type 4 and 5 .

The pair distribution functions $g(4,8)$ depicted in figure 16 at strain values of 8,9 , and $10 \%$ show that the first peak in $g(4,8)$ at about $1.75 \AA$ for less or equal to $8 \%$ strain represents the interface phase $\mathrm{D} 1$ with $\mathrm{N}(4)$ atoms directly on top of $\mathrm{Si}(8)$ atoms. This peak splits into a double peak at $9 \%$. The appearance of an additional peak at about $2.5 \AA$ suggests the fact that part of the system underwent a phase transition into the interface phase D2.
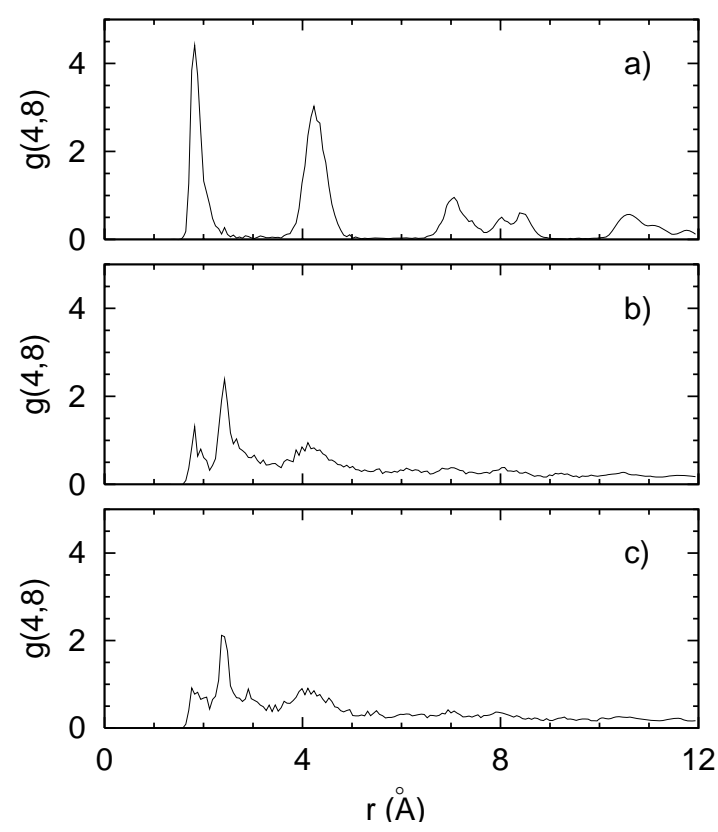

Figure 16: Si-N pair distribution functions $g(4,8)$ across the interface at $600 \mathrm{~K}$ at three different values of strain: (a) $8 \%$, (b) $9 \%$, and (c) $10 \%$. 
In figure 17 the peak heights for the first double peaks of the pair distribution functions $g(4,8)$ are plotted versus externally applied strain for $300 \mathrm{~K}, 600 \mathrm{~K}$, and $900 \mathrm{~K}$. All three temperatures exhibit the phase transition at the same percentage of strain, namely $9 \%$ strain. Throughout the remainder of the simulations the two interface phases coexist in the system.

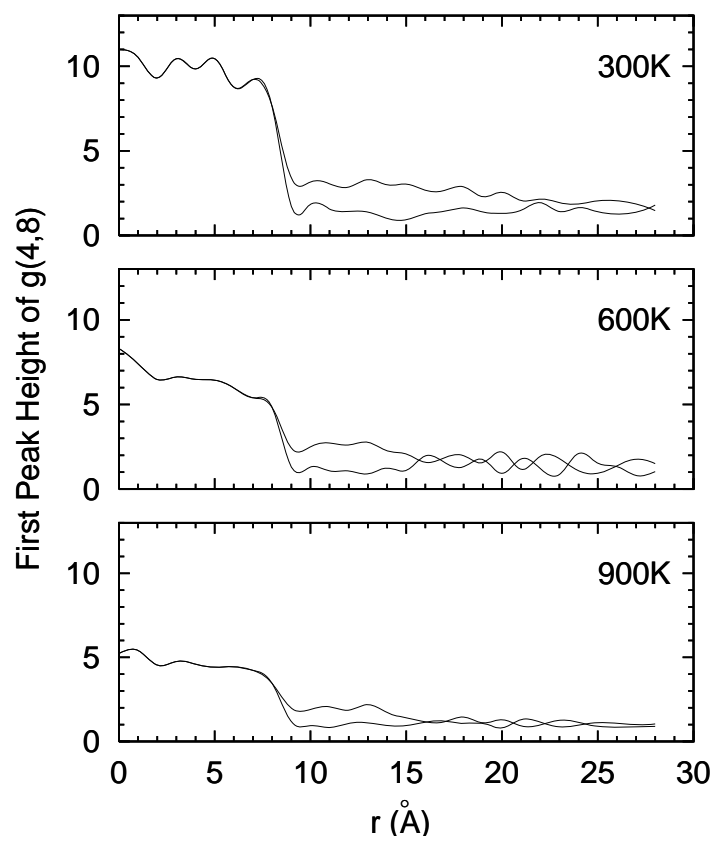

Figure 17: Height of the first (double) peak(s) of $g(4,8)$ across the interface (as depicted in figure 16) at different temperatures. The second peak emerges at $9 \%$ for all three temperatures.

Overall, the following types of failure are observed. At about $8 \%$ strain a crack opens in silicon nitride on the left, at about $9 \%$ another one opens in silicon nitride on the right side of the sample breaking off a centerpiece from the remainder of the silicon nitride film. Slip in Stillinger-Weber silicon releases stress in the substrate. At about $16 \%$ a pit in silicon below the silicon nitride centerpiece becomes visible [Bachlechner et al., 2006]. As continued strain is applied the pit grows in both vertical and horizontal directions (see figure 18). 

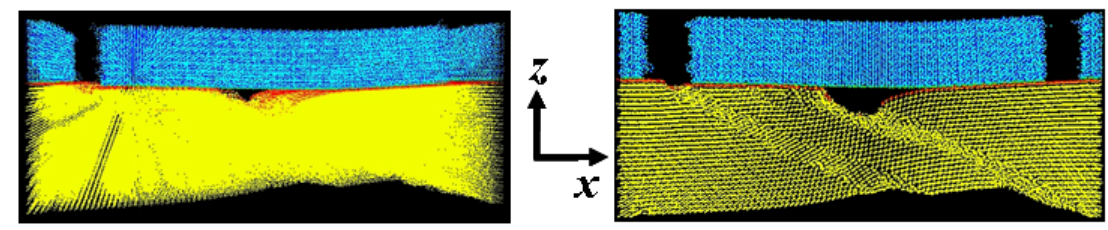

Figure 18: Visualization of the silicon/silicon nitride interface at an externally applied tensile strain of $23 \%$ strain depicting the two cracks in silicon nitride and the slip and pit in silicon. Left: Entire system. Right: Slice in y-direction to better convey the features discussed. 


\section{CONCLUSION}

Molecular-dynamics simulations of large-scale silicon/silicon nitride interfaces were performed to investigate the failure mechanisms when the system is subjected to external tensile strain parallel to the interface. The silicon/silicon nitride interface is modeled as an eight-component system, which allows calculating 36 different selective pair distribution functions. The height of the first peak of the Si-N pair distribution function in bulk silicon, $g(1,3)$, reflects the fact that the major part of the silicon nitride film returns to the almost unstretched configuration after fracture at $8 \%$ strain. Pair distribution functions $g(4,8)$ describe distances across the interface between $\mathrm{N}$ atoms in silicon nitride and Si atoms in silicon. The fact that the first peaks in these functions split into double peaks at $9 \%$ indicate that part of the system undergoes a phase transition from the original interface phase D1 to another local minimum energy interface phase D2.

An additional peak at a smaller distance in the Si-Si pair distribution function at the interface, $g(7,7)$, suggests that originally unbonded $\mathrm{Si}$ atoms form bonds at $14 \%$. From visualization and displacement analysis we have found that a pit forms at $16 \%$. The pair distribution function $g(7,7)$ indicates the onset of the pit already at $14 \%$.

When simulating larger and larger system sizes to investigate the effects of periodic or free surface boundaries, the effects of small system sizes, and the effects of interactions of the longrange stress fields in the system, efficient data analysis is an important issue. Selective pair distribution functions are useful to find points in time in a long-term simulation at which a spatially resolved analysis of properties such as atomic displacements and strains [Schiffbauer et al., 2006], bond lengths and bond angles [ Leonard et al., 2006] involving specific atoms will lead to a more detailed understanding of the underlying atomistic mechanisms of failure. In combination with experimental research, these investigations are important stepping stones in reliably predicting and possibly preventing failure of interfacial systems in general which in turn helps to control electrical properties of devices involving interfaces. 


\section{BIBLIOGRAPHY}

Abraham F F and Gao H 2000 How fast can cracks propagate? Phys. Rev. Lett. 84, 3113

Aberle A G and Hezel R 1996 Advances in low-temperature passivated silicon solar cells Conference Record of the IEEE Photovoltaic Specialists Conference, 371-76

Allen M P 2004 Introduction to Molecular Dynamics Simulation NIC series 23, 1-28

Allen M P and Tildesley D J 1987 Computer Simulation of Liquids Clarendon Press, Oxford

Bachlechner M E, Srivastava D, Owens E T, Schiffbauer J, Anderson J T, Burky M R, Ducatman S C, Gripper A M, Guffey E J, and Serrano Ramos F 2006 Mechanisms of Pit Formation at Strained Crystalline Interfaces Submitted for publication.

Bachlechner M E, Kalia R K, Nakano A, Omeltchenko A, Vashishta P, Ebbsjö I, Madhukar A and Zhao G L 1999 Structural correlations at $\mathrm{Si} / \mathrm{Si}_{3} \mathrm{~N}_{4}$ interface and atomic stresses in $\mathrm{Si} / \mathrm{Si}_{3} \mathrm{~N}_{4}$ nanopixel-10 million-atom molecular dynamics simulation on parallel computers J. Eur. Cer. Soc. 19, 2265-72

Bachlechner M E, Zhang J, Wang Y, Schiffbauer J, Knudsen S R and Korakakis D 2005 Molecular dynamics simulations of the mechanical strength of $\mathrm{Si} / \mathrm{Si}_{3} \mathrm{~N}_{4}$ interfaces Phys. Rev. B 72, 094115$1: 10$

Chelikowsky J R 1988 Transition from metallic to covalent structures in silicon clusters Phys. Rev. Lett. 60, 2669-72

Choudhary D and Clancy P 2005 Application of accelerated molecular dynamics schemes to the production of amorphous silicon J. Chem. Phys. 122, 154509-1:8

Cook S J and Clancy P 1993 Comparison of semi-empirical potential functions for silicon and germanium Phys. Rev. B 47, 7686-99

Deegan R D, Chheda S, Patel L, Marder M, Swinney H L, Kim J, and De Lozanne A 2003 Wavy and rough cracks in silicon Phys. Rev. E 67, 66209-1:7

Demkowicz M J and Argon A S 2005a Liquidlike atomic environments act as plasticity carriers in amorphous silicon Phys Rev B 72, 245205-1:16

Demkowicz M J and Argon A S 2005 Autocatalytic avalanches of unit inelastic shearing events are the mechanism of plastic deformation in amorphous silicon Phys Rev B 72, 24520-1:17

Gerde E and Marder M 2001 Friction and fracture Nature 413, 285-8

Juan Y M, Sun Y and Kaxiras E 1996 Ledge effects on dislocation emission from a crack tip: a firstprinciples study for silicon Phil. Mag. Lett. 73, 233-40

Justo J F, Bazant M Z, Kaxiras E, Bulatov V V and Yip S 1998 Interatomic potential for silicon defects and disordered phases Phys. Rev. B 58, 2539-50

Kalia R K, Nakano A, Omeltchenko A, Tsuruta K and Vashishta P 1997 Role of ultrafine microstructures in dynamic fracture in nanophase silicon nitride Phys. Rev. Lett. 78, 2144 
Kalia R K, Nakano A, Tsuruta K and Vashishta P 1997a Morphology of Pores and Interfaces and Mechanical Behavior of Nanocluster-Assembled Silicon Nitride Ceramic Phys. Rev. Lett. 78, 689-92

Kerr M J, Schmidt J, Cuevas A, and Bultman J H 2001 Surface recombination velocity of phosphorus-diffused silicon solar cell emitters passivated with plasma enhanced chemical vapor deposited silicon nitride and thermal silicon oxide J. Appl. Phys. 89, 3821-6

Kim J W and Yeom H W 2003 Surface and interface structures of epitaxial silicon nitride on Si(111) Phys. Rev. B 67, 035304-1:5

Leonard R H and Bachlechner M E 2006 Bond Angles in the Crystalline Silicon/Silicon Nitride Interface to be published

Li M and Selinger R L B 2003 Molecular dynamics simulations of dislocation instability in a stress gradient Phys. Rev. B 67, 134108-1:6

Lidorikis E, Bachlechner M E, Kalia R K, Nakano A and Vashishta P 2005 Coupling atomistic and continuum length scales in heteroepitaxial systems: Multiscale moleculardynamics/finite-element simulations of strain relaxation in $\mathrm{Si}_{/} \mathrm{Si}_{3} \mathrm{~N}_{4}$ nanopixels Phys Rev B 72, 115338-1:16

Loong C K, Vashishta P, Kalia R K and Ebbsjö I 1995 Crystal structure and phonon density of states of high-temperature ceramic silicon nitride Europhys. Lett. 31, 201-6

Nakano A, Kalia R K, and Vashishta P 1995 Dynamics and morphology of brittle cracks: a molecular-dynamics study of silicon nitride Phys. Rev. Lett. 75, 3138-41

Rambaut C, Jaffrezic $\mathrm{H}$, Kohanoff $\mathrm{J}$ and Fayeulle $\mathrm{S} 1998$ Molecular dynamics simulation of the $\alpha$ $\mathrm{Al}_{2} \mathrm{O}_{3}$ lattice: dynamic propertie Journal of Physics: Condensed Matter 10, 4221-9

Rosselt M, Gao F and Zwicker D 2005 Atomistic study of the migration of di- and tri-interstitials in silicon Phys. Rev. B 71, 245202-1:12

Schiffbauer J E and Bachlechner M E 2006 A Molecular-Dynamics Study of Defects and Failure Mechanisms in Strained Heteroepitaxial Interfaces to be published

Shi X, Shriver M, Zhang Z, Higman T and Campbell S A 2004 Properties of high-k/ultrahigh purity silicon nitride stacks J. Vac. Sci. Technol. A 22, 1146-51

Stillinger $\mathrm{F} \mathrm{H}$ and Weber T A 1985 Computer simulation of local order in condensed phases of silicon Phys. Rev. B 31, 5262-71

Stollwerck G, Reber S and Häßler C 2001 Crystalline Silicon Thin-Film Solar Cells on Silicon Nitride Ceramic Substrates Adv. Mater. 13, 1820-24

Tersoff J 1986 New empirical model for the structural properties of silicon Phys. Rev. Lett. 56, 632-5

Tsuruta K, Nakano A, Kalia R K and Vashishta P 1998 Dynamics of consolidation and crack growth in nanocluster-assembled amorphous silicon nitride J. Am. Ceram. Soc. 81, 433-36

Toby B H and Egami T 1992 Accuracy of pair distribution function analysis applied to crystalline and non-crystalline materials Acta Crystallographica, Section A (Foundations of Crystallography) A48, $336-46$ 
Tuckerman M, Berne B J and Martyna G J 1992 Reversible multiple time scale molecular dynamics J. Chem. Phys. 97, 1990-2001

Vashishta P, Kalia R K, de Leeuw S W, Greenwell D L, Nakano A, Jin W, Yu J, Bi L and Li W 1994 Computer simulation of materials using parallel architectures Computational Materials Science 2, 180-208

Vashishta P, Kalia R K, and Ebbsjö I 1995 Low-energy floppy modes in high-temperature ceramics Phys. Rev. Lett. 75, 858-61

Vashishta P, Kalia R K, Nakano A, Li W and Ebbsjö I 1996 Molecular Dynamics Methods and Large-scale Simulations of Amorphous Materials" in Amorphous Insulators and Semiconductors, M.F. Thorpe and M.I. Mitkova, Editors. NATO ASI p 151

Vashishta P, Nakano A, Kalia R K and Ebbsjö I 1996a Crack propagation and fracture in ceramic films-million atom molecular dynamics simulations on parallel computers Mat. Sci. Eng. B 37, 56-71

Vesely F J 2001 Computational Physics, An Introduction, $2^{\text {nd }}$ Edition Kluwer Academic/Plenum Publishers, New York, Boston, Dordrecht, London, Moscow

Zhakhovskii V V, Zybin S V, Nishihara K and Anisimov S I 1999 Shock wave structure in LennardJones crystal via molecular dynamics Phys. Rev. Lett. 83, 1175-8

Zhao G L and Bachlechner M E 1997 Electronic structure, charge distribution, and charge transfer in $\alpha-$ and $\beta-\mathrm{Si}_{3} \mathrm{~N}_{4}$ and at the $\mathrm{Si}(111) / \mathrm{Si}_{3} \mathrm{~N}_{4}(001)$ interface Europhys. Lett. 37, 287-92

Zhao G L and Bachlechner M E 1998 Electronic structure and charge transfer in $\alpha$ - and $\beta-\mathrm{Si}_{3} \mathrm{~N}_{4}$ and at the $\mathrm{Si}(111) / \mathrm{Si}_{3} \mathrm{~N}_{4}(001)$ interface Phys. Rev. B 58, 1887-9 